2014-06

\title{
Dynamic social networks in guppies (Poecilia reticulata)
}

Wilson, Alexander

http://hdl.handle.net/10026.1/11471

10.1007/s00265-014-1704-0

Behavioral Ecology and Sociobiology

Springer Science and Business Media LLC

All content in PEARL is protected by copyright law. Author manuscripts are made available in accordance with publisher policies. Please cite only the published version using the details provided on the item record or document. In the absence of an open licence (e.g. Creative Commons), permissions for further reuse of content should be sought from the publisher or author. 


\title{
Dynamic social networks in guppies (Poecilia reticulata)
}

\author{
Alexander D. M. Wilson • Stefan Krause • Richard James • \\ Darren P. Croft • Indar W. Ramnarine • Karoline K. Borner • \\ Romain J. G. Clement • Jens Krause
}

Received: 10 December 2013 / Revised: 26 February 2014 / Accepted: 27 February 2014 / Published online: 14 March 2014

(C) Springer-Verlag Berlin Heidelberg 2014

\begin{abstract}
One of the main challenges in the study of social networks in vertebrates is to close the gap between group patterns and dynamics. Usually scan samples or transect data are recorded to provide information about social patterns of animals, but these techniques themselves do not shed much light on the underlying dynamics of such groups. Here we show an approach which captures the fission-fusion dynamics
\end{abstract}

Communicated by J. Lindström

$\mathrm{ADM}$ Wilson and S Krause are shared first authors

Electronic supplementary material The online version of this article (doi:10.1007/s00265-014-1704-0) contains supplementary material, which is available to authorized users.

A. D. M. Wilson · K. K. Borner · R. J. G. Clement · J. Krause Department of the Biology and Ecology of Fishes, Leibniz Institute of Freshwater Ecology and Inland Fisheries, 12587 Berlin, Germany

\section{S. Krause}

Department of Electrical Engineering and Computer Science, Lübeck University of Applied Sciences, Lübeck, Germany

R. James

Department of Physics, University of Bath, Bath BA2 7AY, UK

D. P. Croft

Centre for Research in Animal Behaviour, College of Life and Environmental Sciences, Washington Singer Labs, University of Exeter, Perry Road, Exeter EX4 4QG, UK

I. W. Ramnarine

Department of Life Sciences, University of the West Indies, St Augustine, Trinidad and Tobago

J. Krause

Humboldt University, Faculty of Life Sciences, Berlin, Germany

A. D. M. Wilson $(\bowtie)$

Department of Biology, Carleton University, Ottawa K1S 5B6, Canada

e-mail: alexander.wilson@ymail.com of a fish population in the wild and demonstrates how the gap between pattern and dynamics may be closed. Our analysis revealed that guppies have complex association patterns that are characterised by close strong connections between individuals of similar behavioural type. Intriguingly, the preference for particular social partners is not expressed in the length of associations but in their frequency. Finally, we show that the observed association preferences could have important consequences for transmission processes in animal social networks, thus moving the emphasis of network research from descriptive mechanistic studies to functional and predictive ones.

Keywords Social network analysis · Behaviour type · Fission-fusion dynamics

\section{Introduction}

Networks have become a popular approach to study the social fine structure of animal groups or populations in recent years (Krause et al. 2007; Croft et al. 2008; Wey et al. 2008). There is an increasing realisation that for the investigation of patterns of cooperation, mate choice or dominance hierarchies, we need to consider the complex relationships of multiple individuals that interact with many different conspecifics over time and for which the social network approach provides an ideal framework (Croft et al. 2006; Santos et al. 2006; Sih et al. 2009). Networks have been used as both descriptive and, to a lesser extent, predictive tools to better understand the social organisation of many different taxonomic groups [e.g. social insects: (Naug 2008; Sendova-Franks et al. 2010); reptiles: (Godfrey et al. 2009); teleost fishes: (Croft et al. 2004; Pike et al. 2008; Croft et al. 2009); sharks: (Guttridge et al. 2011); birds: (McDonald 2007; Oh and Badyaev 2010); cetaceans: (Lusseau 2003; Williams and Lusseau 2006); pinnipeds: 
(Wolf et al. 2007); primates: (Flack et al. 2006; Henzi et al. 2009a; Ramos-Fernandez et al. 2009); bats: (Kerth et al. 2011); ungulates: (Sundaresan et al. 2009); rodents: (Lea et al. 2010); herpestids: (Madden et al. 2009)]. Currently, however, three major issues hamper the use of the network approach.

First, in many biological systems we are not only interested in whether an interaction between individuals occurs but also how often it occurs and who initiated it. Typical network metrics such as path length and cluster coefficient, however, become difficult to interpret biologically if we take interaction frequency and directedness into account (Croft et al. 2008). Furthermore, the effect of missing individuals is largely unknown in network studies and as such, it can be difficult to interpret network metrics if 5 or $10 \%$ of the group or population are not observed (Marschall 2007; Perreault 2010; Croft et al. 2011b; Cross et al. 2012). Given the interconnected nature of a network even a few missing individuals that occupy important positions (e.g. very central ones) can change the properties of the entire network.

Second, in order to better understand the evolution of social systems it would be highly desirable to make comparisons between networks (for example, of populations that have evolved under different ecological conditions). However, it turns out that it is difficult to make meaningful comparisons if the networks have different numbers of individuals and interactions between them (Croft et al. 2008). Unfortunately under field conditions (where group size and interaction frequency cannot be controlled), this is often the case.

Finally, other long-standing issues with the social network approach are the problems of how best to sample them and how to relate pattern and dynamics (Croft et al. 2008; Franks et al. 2009; Krause et al. 2009). Most network studies on vertebrates are based on relatively infrequent surveys (ungulates: Sundaresan et al. 2007), transects (dolphins: Lusseau 2003), scan samples of individual associations [primates: (Henzi et al. 2009b), scan sampling every 30 min (RamosFernandez et al. 2009)] and once-per-day captures (fish: Croft et al. 2004). Sampling at such a coarse-grain temporal scale can provide some information regarding social patterns but sheds little light on the underlying dynamics (which generate the patterns). The dynamics, however, are crucial for developing an understanding of processes such as cooperative behaviours or disease transmission that take place on time scales of just minutes or seconds. Furthermore, in most studies on social networks the information gleaned from coarse-grain temporal sampling is then accumulated to produce networks, therein losing the details of internal social dynamics. The reason for the convention of such coarse-grain sampling can in some cases be traced back to the randomisation approach developed by Manly (1995), which is used for data analysis and requires independence of observations (Sundaresan et al. 2007), but in other cases it is due to the difficulty of making continuous field observations.

Here, we describe an approach which is based on Markov chains (MC) that has the potential to circumvent some of the above problems with the network approach. In a MC the future state of a system depends solely on its current state (although higher order MCs can take finite state histories into account). Similarly, we selected MCs because they represent the simplest possible form of an individual-based model. MCs have been used frequently in animal behaviour studies to identify social patterns (e.g. Cane 1978; Metz et al. 1983; Harcourt et al. 2010). However, the main aim of this study was to bridge the gap between pattern and dynamics of social networks by developing a MC model of short-term social interactions that can be used to (a) investigate the social organisation of fish (i.e. Trinidadian guppy, Poecilia reticulata) and (b) act as a blueprint for studies of social networks in other species. We used focal follows of individual fish whose nearest neighbour we recorded every $10 \mathrm{~s}$ over a period of $1.5 \mathrm{~min}$ and thereby built up information on the finescale temporal association patterns of fish in the wild. From this information we developed a MC model of the social dynamics of guppies which allowed us to characterise their fission-fusion behaviour and social network structure (in theory, however, this approach could also be used to add other behaviours such as foraging, sexual and cooperative behaviours). Furthermore, we used this information to explore what impact the social dynamics could potentially have on important transmission processes (e.g. disease or information). Finally, the sampling process we described above (i.e. frequent recording of associations of focus individuals to develop MCs) is particularly suitable for (a) species with small to medium group sizes, (b) where focal follows of individuals are possible in the wild and (c) laboratory studies on social dynamics in general.

\section{Methods}

We caught and marked, using fluorescent elastomer (Croft et al. 2004), all adult female guppies ( $P$. reticulata) $(N=18)$ in a pool (approx. $47.5 \mathrm{~m}^{2}$ ) of the Lower Turure river, Trinidad $\left(10^{\circ} 40^{\prime} 51.5^{\prime \prime} \mathrm{N} 61^{\circ} 10^{\prime} 4.5^{\prime \prime} \mathrm{W}\right)$. Predation in this section of the river is mainly attributable to the presence of large piscivorous predators (e.g. Hoplias malabaricus) in the area, including within our observation pool.

Quantifying behavioural and environmental variables in the wild

Due to intrinsic difficulties associated with being able to locate and track all marked individuals during the same observation day or period, we recorded phenotypic attributes of all 
individually marked guppies only once to allow for a better estimate of each trait for our model (see below). Observations on different days may not have included all individuals and would likely have included variance in environmental conditions, perhaps resulting in the inclusion of an unknown bias to our calculations due to differences in behaviour. At the onset of each 10-min observation period, a focal individual was selected, and a range of behavioural and environmental attributes were characterised. Behaviourally, we recorded activity, shoaling tendency and foraging. Activity was characterised as the total time spent active (s) over the observation period. Time spent holding position in the water column or at rest on the bottom was not included in the activity measurement. Shoaling tendency was quantified as the total time spent within four body lengths of a conspecific female during the observation period (Krause and Ruxton 2002). Time spent in close proximity to a conspecific male was not included in our measure of shoaling tendency due to potential confounding effects associated with mating attempts by males. We quantified foraging behaviour as the total number of feeding attempts made by a focal fish, as evidenced by actual bites on substrate, during the observation session. We also recorded the number of mating attempts by males which was characterised by the total number of copulation attempts (gonopodium thrusts) made by males on the focal female during the observation session. All behaviours were observed simultaneously by three observers. Each observer was responsible for the same phenotypic attribute for all fish.

We quantified two environmental aspects of each focal individual's behaviour: maximum depth and total area travelled during the observation session. Following the completion of each observation session, the maximum water depth for each focal fish was recorded as well as the total area covered $\left(\mathrm{m}^{2}\right)$. Total area was quantified by measuring the maximum length and width travelled within the pool by each fish during the observation session.

\section{Social interaction: data collection}

We focused our attention on a location within the observation pool where fish naturally aggregated (i.e. a social 'hotspot', $5.4 \mathrm{~m}^{2}$ ). The hotspot occupied about $11 \%$ of the total area of the pool but contained on average $40 \%$ of all fish at any given time. We collected information on conspecific associations by following fish of known identity for $90 \mathrm{~s}$ and by recording the identity of their nearest neighbour every $10 \mathrm{~s}$, where applicable. A fish was regarded as having no nearest neighbour if there was no conspecific present within four body lengths of itself during a given observation point. Given the small size of the hotspot and the density of fish, $10 \mathrm{~s}$ was deemed an appropriate time interval because a fish could switch partners within a few seconds at any time. In addition, previous work on a different guppy population had shown that focal individuals encounter conspecifics every $14 \mathrm{~s}$ (Croft et al. 2003) which would provide opportunities for switching partners at a rate lower than our observation frequency. Once we had concluded the 1.5-min focus period for a given marked fish, we identified and tracked a new marked individual (that had not yet been observed as a focus fish) until all marked fish present in the hotspot had been observed. Once an observation period was completed in this way, we gently cleared the hotspot of all fish by using a large branch and subsequently left the hotspot unobserved for $10 \mathrm{~min}$. This was done to make certain that our subsequent observations of the hotspot were independent of each other (but not result in major disruption of the fish or observation environment, see Results). Following this wait period, we then started collecting data for a new network as above. We repeated this process 6 times in a day from about 9.00 a.m. to 2.00 p.m. We had 5 such observation days which were spread over a period of 15 days. The number of marked fish that we observed during a given observation period varied from 5 to 11 fish. From the data on focal fish follows we constructed a fission-fusion model (see below).

\section{Markov chain}

\section{Models}

We constructed 3 models of increasing degree of detail using a data-driven approach (i.e. we tried to build the simplest possible models that explain our observations and made assumptions only when necessary). These models, described as the 'simple model', the 'more detailed model' and the 'individual specific model', are explained in the next paragraphs.

Simple model In this first step of our analyses we sought to describe the general social behaviour of our fish by looking at the patterns common to all focal individuals. If we do not distinguish between individuals, our observation sequences can be written in an abstract way using the symbols $x$ and $i$ to indicate whether the focal fish was alone (no conspecific within four body lengths) or whether it was social (with a conspecific). For example, the sequence $x, x, i, i, i, x$, represents an observation session where the focal fish was initially swimming around on its own for two successive points in time, then with a neighbour for the next three time points and lastly, on its own for the last time point.

In principle, a very simple model for these types of sequences could be constructed that consists of a single parameter, the probability $p(i)$ of observing $i$ at the next time point. This model, however, makes the unlikely assumption that the behaviour at a time point is independent of the behaviour at preceding time points. As such, this very simple model could not explain our observations (Fig. S1 in the Supplementary material). We therefore extended our model by using conditional probabilities $p(i \mid x)$ and $p(i \mid i)$ such that the behaviour at 
the next time point depended on the individual's current behaviour. The process that generates the $x / i$-sequences can then be regarded as a (first-order) MC, where $x$ and $i$ are the states of the MC, and the probability of going from state $x$ to state $i$ is given by $p(i \mid x)$. In the below section regarding the goodness of fit for our models, we show that this modified 'simple model', which consists of only two parameters [since $p(x \mid i)=1-p(i \mid i)$ and $p(x \mid x)=1-p(i \mid x)]$, can explain our observations of $x / i$-sequences. The simple MC model is shown in Fig. 1a, where the probability of going from state $i(x)$ to state $x$ (i) is denoted by $p_{2}\left(p_{3}\right)$.

More detailed model In a sequence of $i$ 's (i.e. a period where the focal fish is consistently social), the nearest neighbour to the focal fish may change from one observation to another. This more detailed model takes this factor into account and extends the simple model by distinguishing between different nearest neighbours. This means, the state $i$ of the simple model is split up into sub-states $i_{1} \ldots i_{k}$, where $k$ is the number of potential nearest neighbours of the focal fish. The resulting model can then describe sequences like $x, x, i_{2}, i_{2}, i_{2}, i_{5}, x$, for example, where the focal fish, after initially swimming around on its own, had individual 2 as its nearest neighbour for three time points and then individual 5 for just one time point.
As above, we again tried to find general patterns common to all individuals. Along these lines, we found that a more detailed model could be constructed by adding a single parameter to the simple model that specified the act of switching between different nearest neighbours. This was possible because the mean time a focal individual spent with a certain nearest neighbour per contact phase did not depend on the neighbour's identity. This means that although the state $i$ of the simple MC model is replaced by $k$ states $i_{1} \ldots i_{k}$ in the more detailed MC model, only the probability for retaining the current nearest neighbour needs to be added (which is the same for $i_{1} \ldots i_{k}$ ), while the other probabilities can be carried over from the simple $\mathrm{MC}$ model.

The more detailed MC model is shown in Fig. 1b, where the probability of retaining the same nearest neighbour is denoted by $q_{1}$. When the contact with this neighbour ends, a new neighbour is chosen among the remaining $k-1$ neighbours with equal probabilities, where the sum of these probabilities and $q_{1}$ must equal $q_{2}$ (the probability of retaining the social state). The parameters of this model (i.e. the transition probabilities $q_{1}, p_{2}$ and $p_{3}$ ) were determined by a maximumlikelihood estimation (see Supplementary material). We used the data from all sessions for this estimation and constructed a time-homogeneous MC model (i.e. we assumed that the
Fig. 1 Egocentric fission-fusion model of social behaviour in the guppy. a A fish can either be social (with a conspecific) denoted by $i$ or alone (no conspecific within 4 body lengths) denoted by $x . p_{2}$ and $p_{3}$ indicate the respective probabilities for changing states and $q_{2}=1-p_{2}$ and $q_{3}=1-p_{3}$ those for retaining current states. $\mathbf{b} \mathrm{A}$ detailed model of state $i$ in the presence of $k$ potential nearest neighbours. An individual is in the sub-state $i_{g}$ of $i$, if individual $g$ is its nearest neighbour. If a focal individual has chosen a nearest neighbour it stays with it with probability $q_{1}$ a

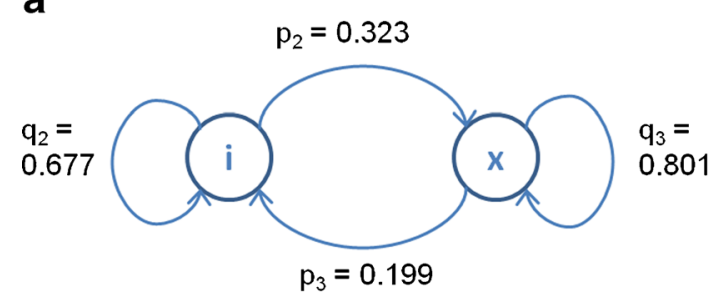

b

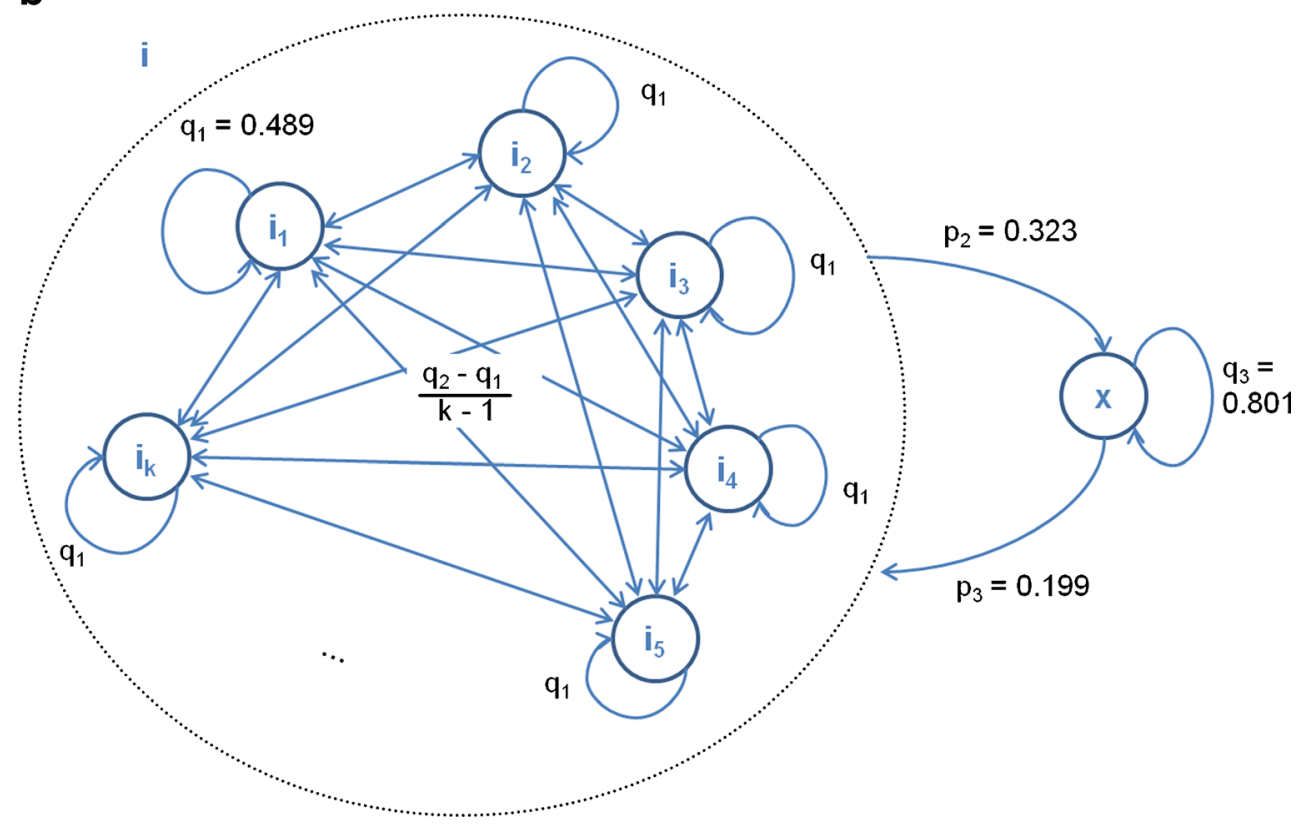


probabilities did not change between the sessions which is justified by the goodness of fit of our model). Since this model uses the same probabilities for all individuals, it can be used for our study system as a null model of no personal preferences between pairs of individuals.

Individual specific models Although the length of the contact phases in our observations followed a global pattern, we found that preferences differed between individuals regarding the number of contact phases. More precisely, when going from state $x$ to state $i$ or when switching to a different neighbour, the focal individual 'picked' a neighbour with individual-specific probabilities.

Based on our detailed model (see above) we constructed individual-specific models for our study system, wherein the focal individual picks neighbours according to the observed preferences. The preference of an individual for another individual was measured by the overall number of contact phases of these individuals divided by the number of sessions where both individuals were present. The probabilities of choosing particular nearest neighbours were then distributed proportionally to these preferences. The probabilities $p_{1}, p_{2}$ and $p_{3}$ of the detailed model, which do not depend on individual preferences, remained unchanged.

Goodness of fit Based on the observations of individual fish we constructed a simple and a more detailed egocentric fission-fusion model (Fig. 1a, b, respectively) as described above. Simulations based on our models reproduced the observed distributions of the lengths of social contact and of contact with a particular nearest neighbour very accurately (Fig. 2). Figure 2d demonstrates another aspect of the goodness of fit of our model by showing that the observed frequencies of being social lie within the model's $90 \%$ tolerance intervals for all 10 time points. Furthermore, after adding individual preferences to these general models the resulting individual specific models very accurately reproduced the observed values of certain network measures (degree and $Y$ measure) as is shown in the results.

\section{Tests}

We performed different Monte Carlo tests using either randomisations of our observations or simulations based on our models (see Supplementary material for a detailed description). For most tests we used simple test statistics which are specified in the results. However, in our analysis of assortment by behavioural traits or attributes we computed the percentage of contact phases each focal fish had with other fish that were similar to it regarding a given trait or attribute. The weighted sum of these percentages determined the value of the test statistic, where the weights were defined by the number of times a fish was observed as focal fish. The similarity was defined using a tolerance level $t$. For example, for a tolerance level of 0.1 and the attribute 'body length', all fish were regarded as similar to the focal fish that had a body length that did not deviate by more than $10 \%$ from the body length of the focal fish. Given that there are individual preferences (see Results section), we used different tolerance levels to determine the range of significant assortments for each behavioural trait or attribute that can explain the preferences. There is no clear criterion for the size that the range of significant tolerance levels should have in order to regard an attribute as an explaining factor. As such, we decided that the corresponding range of percentages for individuals being regarded as similar should be at least 10 .

\section{Results}

The guppy social network which is obtained when combining observations from all 5 days is shown in Fig. 3. Some pairs of fish interacted very frequently (up to 78 times), whereas about $20 \%$ were never seen together (of the latter only $2.9 \%$ are due to the fact that the fish were never observed together at the hotspot at the same time).There was no tendency for particular pairs of fish to be present at the hotspot at the same time (Markov chain Monte Carlo test, $N=10^{8}$, test statistic sum of squares of numbers of co-occurrences, $p=0.062$ ). Fish spent about $40 \%$ of their time being social (i.e. associating with one or more conspecifics), and the average length of social contact (which includes 1.6 different nearest neighbours on average) lasted about $31 \mathrm{~s}$. The average length of contact with a particular nearest neighbour was $19.6 \mathrm{~s}$.

\section{Social dynamics}

Using the more detailed model (with equal transition probabilities for all individuals) as a null model to perform a Monte Carlo test, we found that there were no significant differences among fish in their lengths of social contact (model-based Monte Carlo test, $N=10,000$, test statistic variance of mean lengths of contact, $p=0.292$ ). This means that the model does not require individual-specific transition probabilities to predict the individuals' lengths of social contact.

Based on the theory of Markov chains, our model predicts that after entering a hotspot containing 10 fish, in the absence of any individual preferences, it would on average take $145 \pm$ 60 time steps (approx. $24.1 \pm 10.0 \mathrm{~min}$ ) until all 10 individuals had been the nearest neighbour of the focal fish at least once.

An analysis of the association strength (i.e. number of contacts between individuals) using the above model showed that individuals have preferred nearest neighbours (modelbased Monte Carlo test, $N=10,000$, test statistic sum of squares of relative association strengths, $p=0.0001$ ) regardless of whether the association strength is measured in terms of the 
Fig. 2 Frequency distributions of a the lengths of contact with a particular nearest neighbour, $\mathbf{b}$ the lengths of social contact, i.e. the numbers of successive times a focal individual stayed next to some nearest neighbour, and $\mathbf{c}$ the lengths of being alone in the observed data (grey diamonds) and in the simulation of a MC (black circles). d Observed (grey diamonds) and theoretically expected (black circles) frequencies of state $i$ (social) at each time point within the focus periods of $90 \mathrm{~s}$. The grey bars mark the 5 and $95 \%$ percentiles of the expected frequencies. The theoretic values were computed based on the observed states at time point 1 a

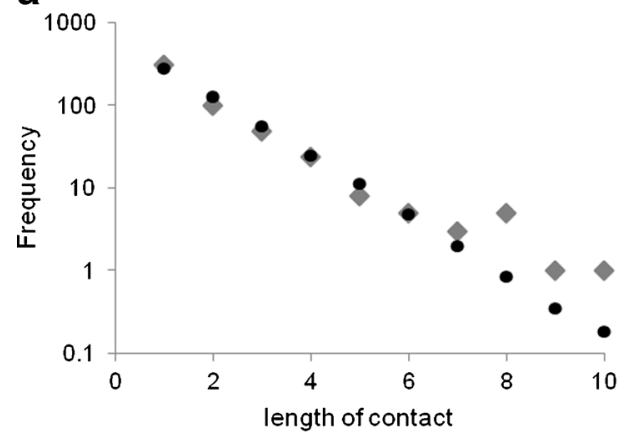

C

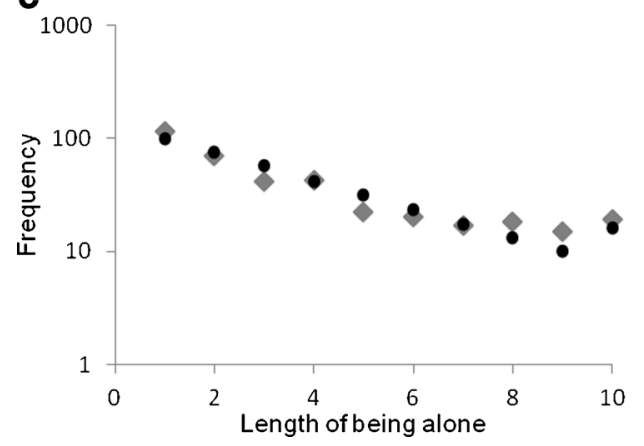

b

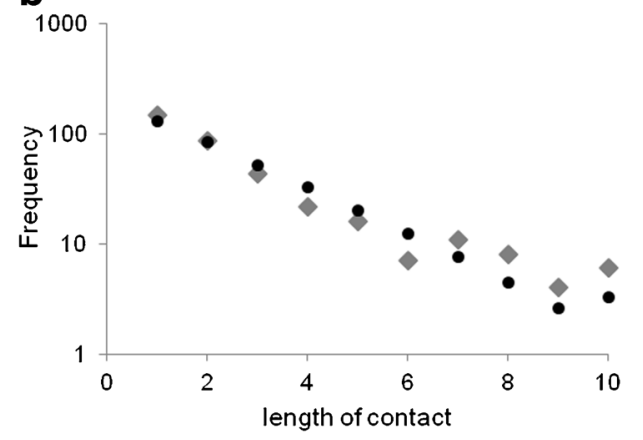

d

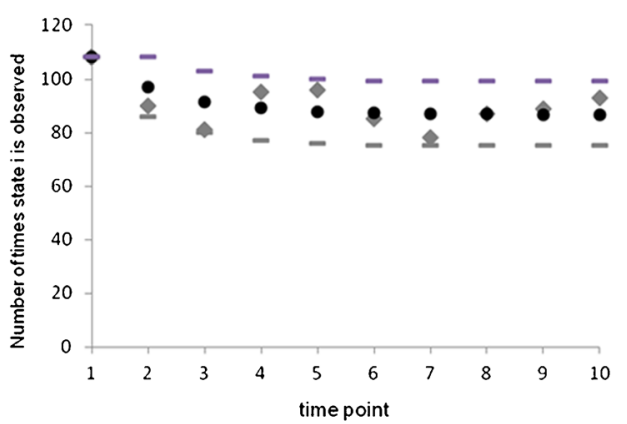

cumulated durations of social contact (i.e. the sum of lengths of successive 10-s intervals with the same partner; Fig. 3a) or whether each succession of contacts with the same partner is regarded as a single contact phase (Fig. 3b). This suggests that the relevant information for preferred conspecifics is not contained in the duration of social contact but in the number of times an individual is chosen as nearest neighbour.

A more detailed analysis of the social fine structure of our fish population revealed that guppies were positively assorted by foraging frequency, area use and preferred water depth (Table 1). Body length assortment was also observed which is surprising given the small body length variation between fish (mean $=28 \mathrm{~mm} \pm 0.30 \mathrm{sd}$ ). Two behavioural variables (activity and shoaling) were also recorded. For these variables we found only very small or no significant ranges, which suggests that neither variable explains much of the observed network associations (Table 1). Data on mating attempts by males showed that females of high attractiveness to males were often found together (Table 1).

\section{Generating networks from a dynamic model}

The aim here was to generate networks from our dynamic models and to compare the model output with our field observation of social structure to test whether our models successfully capture the main features of guppy shoaling behaviour.

We constructed two undirected networks, an unweighted one and a weighted one, from all 5 observation days by adding an edge $\left(i_{1}, i_{2}\right)$ between two nodes representing the individuals $i_{1}$ and $i_{2}$, respectively, whenever one of the two individuals was observed to be nearest neighbour of the other. Weights were assigned according to the number of contact phases between the individuals $i_{1}$ and $i_{2}$. To focus on individuals that were found frequently in the hotspot, only the 15 fish that were observed as focal individuals at least three times were included in the networks. This filtering process reduced the amount of observation data by about $3 \%$. The mean degree (i.e. mean number of different neighbours) of the unweighted network was 11.2 and was significantly lower than expected (compared to networks generated by our detailed model without individual preferences, model-based Monte Carlo test, $N=$ 10,000 , test statistic mean degree, $p=0.0003$, mean degree $=$ $12.4 \pm 0.3 \mathrm{sd})$. For the weighted network we computed the $Y$ measure (Boccaletti et al. 2006), which measures the spread of edge weights of a node by computing the sum of squares of the normalised edge weights (i.e. edge weights divided by the node strength). The mean $Y$-measure of the weighted network was 0.138 which was significantly greater than expected (compared to networks generated by our detailed model without individual preferences, model-based Monte Carlo test, $N=10,000$, test statistic mean $Y, p=0.0001$, mean $Y=0.114 \pm$ $0.004 \mathrm{sd})$. The differences between the observed and the simulated values of both the mean degree and the mean $Y$ measure can be explained by the fact that our general model does not take individual preferences into account. However, our individual-specific models (described in the Methods) yielded the same mean degree (model-based Monte Carlo test, 
a

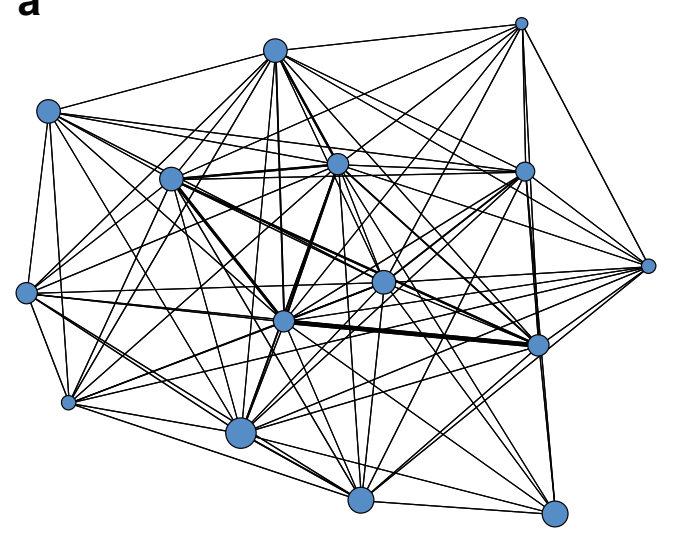

b

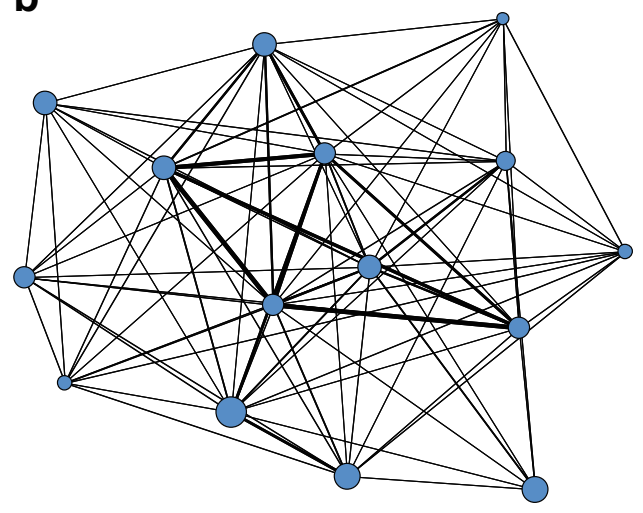

Fig. 3 The social network obtained after combining observations from all 5 days (including 30 observation sessions). In a edge weights are given according to the total number of 10-s intervals at which two individuals were observed as nearest neighbours. Contact frequencies between pairs range from 0 to 78 with a mean of 8.1. In $\mathbf{b}$ consecutive associations were disregarded and the number of contact phases used for edge weights. Contact frequencies between pairs range from 0 to 26 with a mean of 4.5 . Node size corresponds to body size, and spring embedding was used for the layout of the nodes

Table 1 Assortative behaviour within the social network was tested for a number of behavioural traits in the wild. Indicated are the range of tolerance levels (i.e. by how much individuals were different regarding a given behavioural trait) where the test yielded significant $p$ values, the percentage of pairs of individuals that were regarded as similar given the tolerance level and the associated $p$ values. We also included information on mating attempts by males directed towards females in this table

\begin{tabular}{llll}
\hline Behaviour & Tolerance level & $\begin{array}{l}\% \text { individual pairs } \\
\text { within tolerance }\end{array}$ & $p$ value \\
\hline Activity & $0.36-0.42$ & $82-87$ & $0.006-0.046$ \\
Shoaling & - & - & - \\
Foraging & $0.24-1.60$ & $20-85$ & $0.001-0.044$ \\
Area use & $0.28-4.04$ & $17-81$ & $0.001-0.035$ \\
Depth & $0.00-0.10$ & $17-47$ & $0.001-0.042$ \\
Body length & $0.04-0.14$ & $36-68$ & $0.001-0.039$ \\
Mating attempts & $0.66-0.87$ & $41-60$ & $0.001-0.048$ \\
\hline
\end{tabular}

$N=10,000, p=0.474$, mean degree $=11.3 \pm 0.4 \mathrm{sd})$ and $Y$-measure (model-based Monte Carlo test, $N=10,000, p=0.472$, mean $Y=0.139 \pm 0.006 \mathrm{sd}$ ) as the observation. Furthermore, in model-based tests using the individual specific models, none of the above mentioned assortments could be found. This suggests that our individual-specific models capture both the general mechanism that determines the lengths of contact phases and the individual preferences that influence the number of contact phases with particular neighbours.

Predicting disease transmission

Our approach can potentially be used to make predictions about the spread of infectious parasites/diseases or the transmission of socially learnt information. For example, if we had information on how long two fish need to be close to each other to transmit ectoparasites (e.g. Gyrodactylus, see Croft et al. 2011a) to one another, we could make quantitative predictions with our models for the time it would take for an individual to get infected depending on how many conspecifics already carry the ectoparasite. Using our Markov models we carried out a simulation (explained in more detail in the Supplementary material) which makes basic assumptions about the proportion of infected fish in the hotspot and the number of contacts that it takes between two fish for an ectoparasite to be transmitted. We ran this simulation $(N=10,000)$ with and without personal preferences of fish and found that the presence of personal preferences generally increases the time that it takes for an uninfected fish that enters the hotspot to become infected (Fig. 4).

\section{Discussion}

Our approach demonstrates how information gained from direct observations of focal individuals can be used to develop a model which captures the dynamics by which network patterns arise. The two probabilities that characterise our simplest two-state MC were typical of all the fish (and not just some individuals) that visited the hotspot meaning that they identify details of the fission-fusion dynamics which are typical of this population. Likewise we find that the dynamics of how they switch between different social partners is typical of all fish (Fig. 1b). However, when it comes to which fish they have repeated interactions with, we detect 'personal signatures' of individuals which are the result of specific association patterns (Table 1) and potentially also cooperative relationships (Croft et al. 2009). Therefore the MC approach has the potential for developing models of social behaviour that are typical of the individual, the population and the species (for the latter also see Herbert-Read et al. 2013).

In this context another major advantage of the MC approach for generating networks becomes apparent. Missing individuals or differences in observation frequencies between 

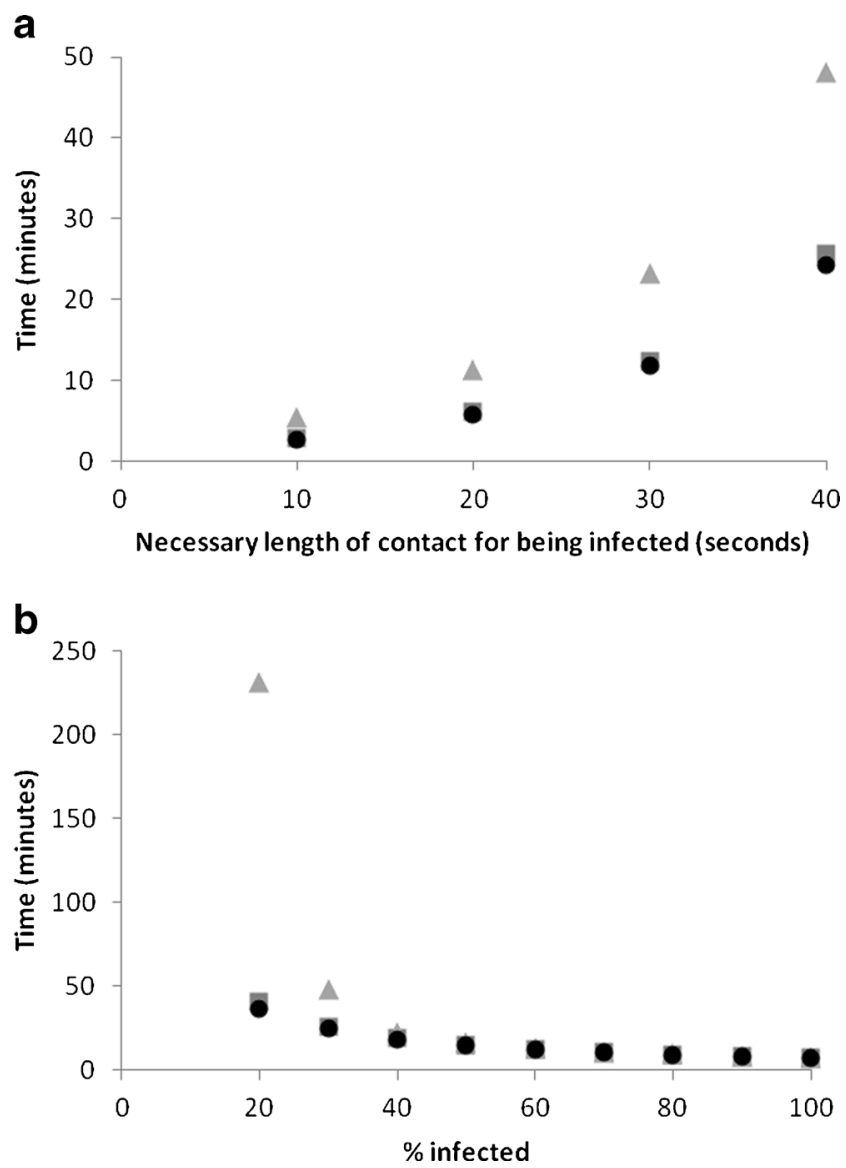

Fig. 4 Mean time it takes for a focal individual to get infected among 10 potential neighbours a as a function of the length of contact necessary for the transmission of a parasite/disease under the assumption that $30 \%$ of the neighbours are infected and $\mathbf{b}$ as a function of the percentage of infected potential neighbours under the assumption that a contact length of $40 \mathrm{~s}$ is necessary for the transmission of a parasite/disease. The curves show simulation results of our model without individual preferences (black circles) and with individual preferences of two actually observed individuals (grey squares and grey triangles) in a session with 11 individuals, i.e. where each focal individual had 10 potential neighbours. The triangles (squares) represent the individual with the highest (lowest) values of all individuals in this session

individuals are often a problematic issue in social network studies and widely discussed in the network literature (Marschall 2007; Cross et al. 2012). Our MC approach shows that at least some aspects of the social dynamics (see Fig. 1) are not individual specific but reflect a more general behaviour or fission-fusion process underlying social networks. This means that missing observations on some individuals have no influence on the MC model (and the networks that are derived from it) because the Markov model captures dynamics that reflect general properties of the social system (and not individual preferences). We showed in our analysis that we can plug the individual preferences of fish as a separate 'module' into the model which then reflects this property of the fish as well and has potentially important consequences for transmission processes in the network.
Our approach of collecting information on focal individuals (i.e. egocentric networks) that are observed with high frequency is particularly suitable to species that live in small to medium groups where individuals can be followed over extended time periods. This might be difficult for most freeranging birds but likely applies to many primate species (Lee 1999), some ungulates and captive animals in general (because groups tend to be small to medium in size and individuals can be seen at all times) where this method would allow a non-invasive, rapid accumulation of data on association patterns in groups or populations.

We observed strong positive associations by a number of different characteristics (foraging, area use, mating, water depth and body length) in the wild. Assortment by foraging and water depth might suggest that individuals of similar metabolic rates associate with others that take similar levels of risk (see also Croft et al. 2009). Similarly, individuals with comparable habitat preferences might require or exploit similar foraging areas which might explain assortment based on area use. Females which were popular with males were often found together. One possibility is that females that preferred shallower water were also exposed to more sexual harassment by males. However, we found no such correlation between water depth and mating attempts.

The Monte Carlo approach which we used for measuring associations by behavioural type uses randomisations but still provides some idea of effect size. Conventional statistics (such as GLMMs) routinely produce effect sizes but create the danger of violating assumptions of independence of data because individuals in networks are interconnected (Croft et al. 2011b). In contrast Monte Carlo-type randomisations which circumvent the problem of dependent data do not normally allow for the calculation of effect sizes in networks (and only give $p$ values). Therefore the approach we show in Table 1 might be useful for future studies on social networks where effect sizes are of interest.

Social preferences (for particular conspecifics) were only detected in terms of the frequency of contact and not its duration between guppies. This result raises the question of whether it is more generally the case in different species. Data on human contact patterns should be readily available in this context, and the question is, for example, whether we talk more often with 'close/important contacts' rather than talk for particularly long periods? Mobile phone and email data should be useful to shed some light on this topic. A study on 94 individuals (who agreed to having their proximity data and calling patterns identified) and their contact patterns found that reciprocal friendships were mainly identified by social context (i.e. time spent together outside work, Eagle et al. 2009).

The example of mobile phone data raises again the issue of directedness and frequency of interactions which we briefly mentioned as a problem in current network analysis. When analysing network patterns with the notion of understanding processes on networks (regarding information or infectious diseases), researchers have often used network metrics such as 
the path length or the cluster coefficient to make predictions (Watts and Strogatz 1998; Krause et al. 2007; Croft et al. 2008). However, once we have identified the interaction dynamics of a social system using MCs, we no longer need these metrics because the MC model can potentially be used to make such predictions much more accurately. It remains a challenge, however, to pick the right observation frequency for individuals to get the time scale of the sampling procedure right to fit the transmission process that we want to model. In the case of our guppies, for example, we made sure to select an observation frequency which is more fine scale than the encounter frequency with conspecifics (Croft et al. 2003).

In Fig. 1a we constructed the simplest MC with just two states (social and solitary) and two transition probabilities. This in itself is already an advance on the conventional measure of shoaling/grouping tendency in a population (Krause and Ruxton 2002) because it allows additional insight into the underlying dynamics of the behaviour. For example, two populations that have the same average shoaling tendency could have different social dynamics because the same level of shoaling tendency can be an outcome of different combinations of transitional probabilities. If we take into account that this approach can be extended to how fish select particular conspecifics (Fig. 1b) then the MC provides a promising tool for making comparisons between populations where differences in ecological factors have selected for different behaviours and life history traits (Magurran 2005). For example, some populations of the shortfin molly, Poecilia mexicana, inhabit both surface and cave habitats (Riesch et al. 2011). Our approach could be used to compare the social networks of both ecotypes to provide new insight into how molly social behaviour on the surface has changed and adapted to a cave environment. In addition, some network comparisons have previously been complicated by the fact that the number of nodes (i.e. individuals) and edges (i.e. connection between individuals) differs between networks (Croft et al. 2008). However, the MC approach shown in this study makes it possible to directly compare probabilities regarding state changes. As a note of caution, we should mention that differences in population density may have an influence on these probabilities. That said, we did not detect such an influence in our data set despite the fact that fish number in the hotspot varied from 5 to 11 (which indicates that our approach is probably robust against small variations in density). Nevertheless we expect that the MC approach will be a promising tool for network comparisons.

Our study was based on the behaviour of female guppies at a local hotspot where most of the interactions between fish in the pool took place. Further study has indicated that our MC approach for modelling social interaction can also be applied to entire pools (i.e. small populations) and is not restricted to hotspots.

The sociological literature contains several examples of analyses of dynamic social networks (see Pinter-Wollman et al. 2013 for a review of approaches in both the social and biological sciences). Sociologists usually study social networks by using questionnaires asking people, for example, who their friends are. The structure of social relationships can thus be quantified directly in humans, and these relationships tend to be stable over time. These features allow for modelling approaches such as stochastic actor-based models to be used to study the temporal dynamics of social relationships (Snijders et al. 2010). In contrast, in studies on animal social networks, relationships and social structure have to be inferred from information on patterns of social associations or interactions. Traditionally these data tend to be aggregated over time, and social structure is quantified as a static network (Croft et al. 2008). This static abstraction, however, is restrictive, as it does not allow us to ask questions relating to the temporal dynamics of structure (Blonder et al. 2012). The approach that we have developed allows us to infer the social structure of the population from the temporal dynamics of the social interactions. Given that self-reporting is restricted to humans and that animal networks are often very dynamic and social interactions short lived, we feel that this approach has great potential for unravelling the social structure and dynamics of nonhuman animals.

As a final point we would like to stress that the aim of this paper was not to prove that the behaviour of fish is Markovian but to demonstrate that simple MCs may capture enough of the fission-fusion dynamics to be a useful tool to make predictions (Fig. 4). Although we did not use the MC approach to its full potential in our own study (by restricting it to shoaling behaviour), it is clear that direct observations on a whole range of behavioural states (e.g., foraging, sexual activity, aggression) can be directly made. This opens up the possibility to create more complex models of behaviour with important implications for processes such as cooperation, sexual selection and information and disease transmission in groups and populations. Regardless of whether our MC approach will be fruitful for other taxonomic groups, it is clear that the conceptual step of closing the gap between dynamics and social patterns (as shown in this article) will be necessary in the future to increase our understanding of social systems. In conclusion the MC approach we developed could be used to move the emphasis of animal social network studies from mechanistic questions to more functional ones, and from description towards prediction.

Acknowledgments ADM Wilson acknowledges financial support from research fellowships from the Alexander von Humboldt foundation and IGB. RC was supported by an IGB studentship, KB by a Nafög studentship and DPC by funding from the Leverhulme Trust. We would like to thank Kharan Deonarinesingh for assistance in the field.

Ethical standards This research was performed in accordance with the laws, guidelines and ethical standards of the country in which they were performed (Trinidad). 


\section{References}

Blonder B, Wey TW, Dornhaus A, James R, Sih A (2012) Temporal dynamics and network analysis. Methods Ecol Evol 3:958-972

Boccaletti S, Latora V, Moreno Y, Chavez M, Hwang DU (2006) Complex networks: structure and dynamics. Phys Rep 424:175-308

Cane V (1978) On fitting low-order Markov chains to behaviour sequences. Anim Behav 26:332-338

Croft DP, Arrowsmith BJ, Bielby J, Skinner K, White E, Couzin ID, Magurran AE, Ramnarine I, Krause J (2003) Mechanisms underlying shoal composition in the Trinidadian guppy, Poecilia reticulata. Oikos 100:429-438

Croft DP, Krause J, James R (2004) Social networks in the guppy (Poecilia reticulata). Proc R Soc Lond B 271:S516-S519

Croft DP, James R, Thomas POR, Hathaway C, Mawdsley D, Laland KN, Krause J (2006) Social structure and co-operative interactions in a wild population of guppies (Poecilia reticulata). Behav Ecol Sociobiol 59:644-650

Croft DP, James R, Krause J (2008) Exploring animal social networks. Princeton University Press, Princeton

Croft DP, Krause J, Darden SK, Ramnarine IW, Faria JJ, James R (2009) Behavioural trait assortment in a social network: patterns and implications. Behav Ecol Sociobiol 63:1495-1503

Croft DP, Edenbrow M, Darden SK, Ramnarine IW, Oosterhout C, Cable $\mathrm{J}$ (2011a) Effect of gyrodactylid ectoparasites on host behaviour and social network structure in guppies Poecilia reticulata. Behav Ecol Sociobiol 65:2219-2227

Croft DP, Madden JR, Franks DW, James R (2011b) Hypothesis testing in animal social networks. Trends Ecol Evol 26:502-507

Cross PC, Creech TG, Ebinger MR, Heisey DM, Irvine KM, Creel S (2012) Wildlife contact analysis: emerging methods, questions, and challenges. Behav Ecol Sociobiol 66:1437-1447

Eagle N, Pentland A, Lazer D (2009) Inferring friendship network structure by using mobile phone data. Proc Natl Acad Sci U S A 106: 15274-15278

Flack JC, Girvan M, de Waal FBM, Krakauer DC (2006) Policing stabilizes construction of social niches in primates. Nature 439: 426-429

Franks DW, James R, Noble J, Ruxton GD (2009) A foundation for developing a methodology for social network sampling. Behav Ecol Sociobiol 63:1079-1088

Godfrey SS, Bull C, James R, Murray K (2009) Network structure and parasite transmission in a group living lizard, the gidgee skink, Egernia stokesii. Behav Ecol Sociobiol 63:1045-1056

Guttridge TL, Gruber SH, DiBattista JD, Feldheim KA, Croft DP, Krause S, Krause J (2011) Assortative interactions and leadership in a freeranging population of juvenile lemon shark Negaprion brevirostris. Mar Ecol Prog Ser 423:235-245

Harcourt JL, Sweetman G, Manica A, Johstone RA (2010) Pairs of fish resolve conflicts over coordinated movement by taking turns. Curr Biol 20:156-160

Henzi SP, Lusseau D, Weingrill T, Schaik CP, Barrett L (2009a) Cyclicity in the structure of female baboon social networks. Behav Ecol Sociobiol 63:1015-1021

Henzi SP, Lusseau D, Weingrill T, van Schaik CP, Barrett L (2009b) Cyclicity in the structure of female baboon social networks. Behav Ecol Sociobiol 63:1015-1021

Herbert-Read JE, Krause S, Morrell LJ, Schaerf TM, Krause J, Ward AJW (2013) The role of individuality in collective group movement. Proc R Soc B 280:2012-2564

Kerth GG, Perony NN, Schweitzer FF (2011) Bats are able to maintain long-term social relationships despite the high fission-fusion dynamics of their groups. Proc R Soc Lond B 278:2761-2767

Krause J, Ruxton GD (2002) Living in groups. Oxford University Press, Oxford
Krause J, Croft DP, James R (2007) Social network theory in the behavioural sciences: potential applications. Behav Ecol Sociobiol 62:15-27

Krause J, Lusseau D, James R (2009) Animal social networks: an introduction. Behav Ecol Sociobiol 63:967-973

Lea AJ, Blumstein DT, Wey TW, Martin JGA (2010) Heritable victimization and the benefits of agonistic relationships. Proc Natl Acad Sci U S A 107:21587-21592

Lee PC (1999) Comparative primate socioecology. Cambridge University Press, Cambridge

Lusseau D (2003) The emergent properties of a dolphin social network. Proc R Soc Lond B 270:S186-S188

Madden JR, Drewe JA, Pearce GP, Clutton-Brock TH (2009) The social network structure of a wild meerkat population: 2 . Intragroup interactions. Behav Ecol Sociobiol 64:81-95

Magurran AE (2005) Evolutionary biology: The Trinidadian guppy. Oxford University Press, Oxford

Manly BFJ (1995) A note on the analysis of species co-occurrences Ecology 76:1109-1115

Marschall N (2007) Methodological pitfalls in social network analysis: Why current methods produce questionable results. VDM, Saarbrücken

McDonald DB (2007) Predicting fate from early connectivity in a social network. Proc Natl Acad Sci U S A 104:10910-10914

Metz HAJ, Dienske H, de Jonge G, Putters FA (1983) Continuous-time Markov chains as models for animal behaviour. Bull Math Biol 45: 643-658

Naug D (2008) Structure of the social network and its influence on transmission dynamics in a honeybee colony. Behav Ecol Sociobiol 62:1719-1725

Oh KP, Badyaev AV (2010) Structure of social networks in a passerine bird: consequences for sexual selection and the evolution of mating strategies. Am Nat 176:E80-E89

Perreault C (2010) A note on reconstructing animal social networks from independent small-group observations. Anim Behav 80:551-562

Pike TW, Samanta M, Lindstroem J, Royle NJ (2008) Behavioural phenotype affects social interactions in an animal network. Proc R Soc Lond B 275:2515-2520

Pinter-Wollman N, Hobson EA, Smith JE, Edelman AJ, Shizuka D et al (2013) The dynamics of animal social networks: analytical, conceptual, and theoretical advances. Behav Ecol. doi:10.1093/beheco/ $\operatorname{art047}$

Ramos-Fernandez G, Boyer D, Aureli F, Vick LG (2009) Association networks in spider monkeys (Ateles geoffroyi). Behav Ecol Sociobiol 63:999-1013

Riesch R, Plath M, Schlupp I (2011) Toxic hydrogen sulphide and dark caves: pronounced male life-history divergence among locally adapted Poecilia mexicana (Poeciliidae). J Evol Biol 24:596-606

Santos FC, Pacheco JM, Lenaerts T (2006) Evolutionary dynamics of social dilemmas in structured heterogeneous populations. Proc Natl Acad Sci U S A 103:3490-3494

Sendova-Franks AB, Hayward RK, Wulf B, Klimek T, James R et al (2010) Emergency networking: famine relief in ant colonies. Anim Behav 79:473-485

Sih A, Hanser SF, McHugh KA (2009) Social network theory: new insights and issues for behavioral ecologists. Behav Ecol Sociobiol 63:975-988

Snijders TAB, van de Bunt GG, Steglich CEG (2010) Introduction to stochastic actor-based models for network dynamics. Soc Networks 32:44-60

Sundaresan SR, Fischhoff IR, Dushoff J, Rubenstein DI (2007) Network metrics reveal differences in social organization between two fissionfusion species, Grevy's zebra and onager. Oecologia 151:140-149

Sundaresan, Fischhoff IR, Dushoff J (2009) Avoiding spurious findings of nonrandom social structure in association data. Anim Behav 77: 1381-1385 
Watts DJ, Strogatz SH (1998) Collective dynamics of 'small-world' networks. Nature 393:440-442

Wey T, Blumstein DT, Shen W, Jordan F (2008) Social network analysis of animal behaviour: a promising tool for the study of sociality. Anim Behav 75:333-344
Williams R, Lusseau D (2006) A killer whale social network is vulnerable to targeted removals. Biol Lett 2:497-500

Wolf JBW, Mawdsley D, Trillmich F, James R (2007) Social structure in a colonial mammal: unravelling hidden structural layers and their foundations by network analysis. Anim Behav 74:1293-1302 